\title{
Bone health after diagnosis of breast cancer
}

\author{
Shannon M. Ruzycki MD, Nancy A. Nixon MD
}

Cite as: CMAJ 2018 December 10;190:E1452. doi: 10.1503/cmaj.180802

1 Treatment of breast cancer may increase fracture risk ${ }^{1}$

Low bone density is an adverse effect of aromatase inhibitors, and fracture rates are highest in patients who receive these inhibitors. ${ }^{1}$ Premenopausal women with breast cancer with high-risk features may have ovarian suppression as part of their treatment, leading to early menopause. ${ }^{1}$ These factors contribute to the 36 fractures for every 1000 women who receive therapy for breast cancer each year. ${ }^{1}$

2 Patients with breast cancer should be assessed for fracture risk using a validated tool

Validated tools for assessing fracture risk include the Fracture Risk Assessment Tool (FRAX) or Canadian Association of Radiologists - Osteoporosis Canada calculator (CAROC) (available at https://osteoporosis.ca/health -care-professionals/tools/). Postmenopausal women with breast cancer, including those with treatment-induced menopause, and patients who require aromatase inhibitors should have a bone density measurement. ${ }^{2}$ Patients with low bone density or high fracture risk should take calcium and vitamin $D$, and be counselled on weight-bearing exercise, resistance training and smoking cessation. ${ }^{2}$

Bone is the most common site of metastases for patients with breast cancer ${ }^{3}$

In the first year after diagnosis, $39 \%$ of patients with metastatic breast cancer to bone develop a skeletal-related event, a composite outcome that includes pathologic fracture, bone pain, need for palliative radiation and hypercalcemia. ${ }^{3}$

Bisphosphonates can reduce morbidity from bone metastases

Two combined randomized trials showed that $51 \%$ of patients with metastatic breast cancer who received adjuvant bisphosphonate therapy had a skeletal-related event compared with $64 \%$ of patients on placebo. ${ }^{3,4}$

Bisphosphonates improve survival and prevent bone metastasis in postmenopausal women with early-stage breast cancer

A meta-analysis of 18766 women randomly assigned to adjuvant bisphosphonate therapy after diagnosis showed a $14 \%$ lower risk of disease recurrence and 3.3\% absolute reduction in 10-year overall mortality for those who had undergone natural or treatment-induced menopause. ${ }^{5}$ These benefits were not seen in premenopausal women, and are expected to be greatest in postmenopausal women who have the highest risk of breast cancer recurrence and lowest risk of bisphosphonate toxicity, including osteonecrosis of the jaw and atypical femur fracture.,5

\section{References}

1. Kristensen B, Ejlertsen B, Jensen MB, et al.; Danish Breast Cancer Cooperative Group. The occurrence of fractures after adjuvant treatment of breast cancer: a DBCG register study. Acta Oncol 2018;57:141-5.

2. Papaioannou A, Morin S, Cheung AM, et al.; Scientific Advisory Council of Osteoporosis Canada. 2010 clinical practice guidelines for the diagnosis and management of osteoporosis in Canada: summary. CMAJ 2010;182:1864-73.

3. Clemons M, Gelmon KA, Pritchard KI, et al. Bone-targeted agents and skeletal-related events in breast cancer patients with bone metastases: the state of the art. Curr Oncol 2012;19:259-68.

4. Lipton A, Theriault RL, Hortobagyi GN, et al. Pamidronate prevents skeletal complications and is effective palliative treatment in women with breast carcinoma and osteolytic bone metastases: long term follow-up of two randomized, placebo-controlled trials. Cancer 2000;88:1082-90.

5. Early Breast Cancer Trialists' Collaborative Group (EBCTCG). Adjuvant bisphosphonate treatment in early breast cancer: meta-analyses of individual patient data from randomised trials. Lancet 2015;386:1353-61.

Competing interests: None declared.

This article has been peer reviewed.

Affiliations: Department of Medicine (Ruzycki), University of Calgary; Department of Oncology (Nixon), Tom Baker Cancer Centre, Calgary, Alta.

Correspondence to: Shannon Ruzycki, sarro@ualberta.ca 